\title{
Barriers, supports, and effective interventions for uptake of human papillomavirus- and other vaccines within global and Canadian Indigenous peoples: a systematic review protocol
}

Kelly J. Mrklas ${ }^{1,2}$, Shannon MacDonald ${ }^{3}$, Melissa A. Shea-Budgell ${ }^{4,5}$, Nancy Bedingfield ${ }^{2}$, Heather Ganshorn ${ }^{6}$, Sarah Glaze ${ }^{5,7}$, Lea Bill ${ }^{8}$, Bonnie Healy ${ }^{8}$, Chyloe Healy ${ }^{8}$, Juliet Guichon², Amy Colquhoun ${ }^{9}$, Christopher Bell ${ }^{9}$, Ruth Richardson ${ }^{10}$, Rita Henderson ${ }^{2}$, James Kellner ${ }^{11}$, Cheryl Barnabe ${ }^{2,11}$, Robert A. Bednarczyk ${ }^{12}$, Angeline Letendre ${ }^{13}$ and Gregg S. Nelson ${ }^{4,5,7^{*}}$

\begin{abstract}
Background: Despite the existence of human papilloma virus (HPV) vaccines with demonstrated safety and effectiveness and funded HPV vaccination programs, coverage rates are persistently lower and cervical cancer burden higher among Canadian Indigenous peoples. Barriers and supports to HPV vaccination in Indigenous peoples have not been systematically documented, nor have interventions to increase uptake in this population. This protocol aims to appraise the literature in Canadian and global Indigenous peoples, relating to documented barriers and supports to vaccination and interventions to increase acceptability/uptake or reduce hesitancy of vaccination. Although HPV vaccination is the primary focus, we anticipate only a small number of relevant studies to emerge from the search and will, therefore, employ a broad search strategy to capture literature related to both HPV vaccination and vaccination in general in global Indigenous peoples.
\end{abstract}

Methods: Eligible studies will include global Indigenous peoples and discuss barriers or supports and/or interventions to improve uptake or to reduce hesitancy, for the HPV vaccine and/or other vaccines. Primary outcomes are documented barriers or supports or interventions. All study designs meeting inclusion criteria will be considered, without restricting by language, location, or data type. We will use an a priori search strategy, comprised of key words and controlled vocabulary terms, developed in consultation with an academic librarian, and reviewed by a second academic librarian using the PRESS checklist. We will search several electronic databases from date of inception, without restrictions. A pre-defined group of global Indigenous websites will be reviewed for relevant gray literature. Bibliographic searches will be conducted for all included studies to identify relevant reviews. Data analysis will include an inductive, qualitative, thematic synthesis and a quantitative analysis of measured barriers and supports, as well as a descriptive synthesis and quantitative summary of measures for interventions.

(Continued on next page)

\footnotetext{
* Correspondence: gsnelson@ucalgary.ca

${ }^{4}$ Arnie Charbonneau Cancer Institute, University of Calgary, Calgary, Canada

${ }^{5}$ Department of Oncology, Cumming School of Medicine, University of

Calgary, Calgary, Canada

Full list of author information is available at the end of the article
} 


\begin{abstract}
(Continued from previous page)
Discussion: To our knowledge, this study will contribute the first systematic review of documented barriers, supports, and interventions for vaccination in general and for HPV vaccination. The results of this study are expected to inform future research, policies, programs, and community-driven initiatives to enhance acceptability and uptake of HPV vaccination among Indigenous peoples.
\end{abstract}

Systematic review registration: PROSPERO Registration Number: CRD42017048844

Keywords: Barriers, Supports, Human papillomavirus (HPV), Vaccination, Indigenous populations, Acceptance, Hesitancy, Uptake, Cancer prevention

\section{Background \\ Rationale}

Persistent infection with human papillomavirus (HPV) strains 16 and 18 can cause cervical and other cancers [1]. The prevalence of these strains can vary by socioeconomic factors, including geography and ethnicity $[2,3]$. There are well-documented inequities in cancer prevention, disease burden, and subsequent treatment, for First Nation (FN), Inuit, and Métis peoples in Canada [2, 4-10]. While acknowledging that there is heterogeneity across and within each of these peoples, we respectfully refer to them 'Indigenous unless otherwise specified. The prevalence of HPV infection and cervical cancer is significantly higher [11-23] and HPV vaccination rates lower among Indigenous peoples [23]. This inequity exists despite the availability of vaccines with demonstrated safety profiles and efficacy, and funded vaccination programs [24-26].

Enhancing HPV vaccination is a potentially high-impact, low-cost opportunity for cancer risk reduction among Indigenous peoples. As part of a tri-phase study in First Nations people to (1) establish baseline HPV vaccination rates, (2) systematically identify and validate the barriers and supports to HPV vaccination, and (3) comprehensively map documented and reported supports and barriers to HPV vaccination to develop a theory-informed, context-appropriate knowledge translation intervention, this synthesis aims to identify documented barriers, supports, and intervention strategies for HPV vaccination in Canadian and global Indigenous populations.

\section{Vaccine acceptability, hesitancy, and uptake}

Despite its success as a public health intervention [27-34], vaccination in many countries still falls short of levels needed to ensure community protection [30, 35-37]. Even where vaccination rates are high, ongoing acceptance remains a vulnerability [35], as evidenced by significant variability among different groups within high vaccinecoverage countries $[30,37]$. Measuring vaccination rates can be challenging, requiring different metrics to quantify impact. "Vaccine coverage" (e.g., the proportion of the target population who have received the vaccine) is the typical population-level measure of protection [30, 37-39], while "vaccine acceptability" and "uptake" are more common, individual-level measures. "Vaccine acceptability" reflects individuals' willingness to accept vaccination, typically assessed as attitudes, beliefs, and/or intentions [40]. A more commonly used term, "vaccine hesitancy", refers to a delay in acceptance or refusal of vaccines despite the availability of vaccine services [41]. "Vaccine uptake" reflects the behavior of getting vaccinated [37]. Understanding the barriers and supports to vaccine acceptability/hesitancy, moving from acceptance to uptake, and uptake from multiple perspectives (e.g., recipient, parents/guardians, health care providers, policy makers) [25], is a critical first step to tailoring strategies to enhance vaccination $[37,42]$.

Barriers and supports to vaccine acceptability and uptake General barriers and supports to vaccine acceptability and uptake of childhood vaccines have been documented $[41,43,44]$. The most common factors include sociodemographic characteristics (e.g., race, marital status, maternal age/education, family size/income) [45-50], knowledge and beliefs (e.g. fear of side effects, lack of perceived susceptibility to disease) [45, 46, 49, 50], trust in the establishment (e.g., trust in the medical community or government) [46, 49-51], social and cultural norms (e.g., family, religious, or community influences) $[45,49]$, worldview regarding health (e.g., preference for natural interventions) [45, 49], healthcare provider influence (e.g., provider recommendations and/or knowledge) $[49,50]$, competing priorities (e.g., social support, child care) $[50,52,53]$, concern about vaccination processes (e.g., vaccination pain or needles) [46, 49-51], and access to services (e.g., clinic hours and location) [48-50]. Some of these factors are more prominent in specific sub-populations and/or for specific vaccines [30, 40].

\section{HPV vaccine-specific barriers, supports, and effective interventions}

In Canada, the HPV vaccine was introduced into national and regional vaccination programs targeting preadolescents in school settings [54], to prevent cancers caused by HPV $[55,56]$. Vaccines preventing HPV infection demonstrate long-term effectiveness and acceptable safety profiles [24]. However, a number of issues specific 
to the HPV vaccine influence its acceptability and uptake in the general population; these factors are well described in systematic reviews $[25,26,57-61]$ and include knowledge and perceived risk of HPV disease, misinformation about vaccine safety, cost, misperceptions that the vaccine induces sexual promiscuity, and multi-dose vaccine series delivery. Additional challenges in the school setting include the information and consent process, vaccination setting, and student anxiety regarding privacy and pain during vaccination [62]. In high-income countries, access to and receipt of the HPV vaccine is determined by factors that influence policy makers, health care professionals, and parental decision-making, including financial constraints, social norms and values concerning sexual activity, and trust in vaccination programs and health care providers [61]. Further, barriers, and supports may be context-specific; for example, access to HPV vaccine may be facilitated by universal coverage health care systems or hindered by parents' cultural or religious perceptions about sexual activity [61]. Much of the literature reports barriers and supports to HPV vaccine acceptability, as reported by parents, health care providers, and policy makers [25], rather than vaccine recipients. This gap in self-reported data hinders effective, evidence- and theoryinformed intervention mapping [63].

A recent systematic review [25] summarized the nature and frequency of adolescent females' self-reported barriers to HPV vaccination. These factors included cost, perceived lack of need, concerns about safety and side effects, perceived efficacy, perceived relative benefit, vaccination logistics, fear of needles, vaccine novelty, perception that sexual inactivity precludes the need for vaccination, negative physician-related recommendations, social norms, inability to discuss the vaccine with parents, lack of awareness or information, anti-vaccination views, subjective norms, pregnancy (or trying to conceive), self-efficacy, mistrust of pharmaceutical companies, and the perception that alternative strategies are available. Cost and the perception that HPV vaccination leads to increased sexual activity were previously identified as vaccination barriers [60]. Adolescent females' self-reported supports to HPV vaccination included perceived benefit, positive health care provider recommendations, social norms, parental endorsement, perceived risk, cost-free access, subjective norms, perceived severity, positive attitude toward vaccine or reported personal importance of vaccine, self-efficacy, and vaccine efficacy $[25,60]$.

Although a recent systematic review reported a lack of consistency and detailed reporting of interventions to increase HPV vaccine uptake [64], it found that the nature and magnitude of effect for national and international strategies (e.g., single and multifaceted behavioral [targeted at patient, provider and patient-provider] and environmental [local and national level policies]) were documented. It also revealed that environmental interventions (e.g., school-based vaccine administration) achieved the highest vaccination rates, but noted a lack of data for differentiating subpopulations. The need was underscored for specific strategies and interventions to target underserved populations, including Indigenous youth $[64,65]$.

\section{Barriers and supports to HPV vaccine acceptability and uptake in indigenous peoples}

HPV vaccine coverage levels in the general population of pre-adolescents may hide sub-populations with lower coverage, including Indigenous peoples who are known to have higher HPV infection rates $[2,11,66]$, lower cervical cancer screening rates [13, 14, 67], higher rates of invasive cervical cancer $[15,68]$, higher rates of cervical cancer-related hospitalizations [16], and lower rates of cervical cancer survival $[17,18,69]$. There is a paucity of data on HPV vaccination coverage in Indigenous peoples; however, it is well known that other childhood vaccinations in these groups are typically below that of the general population [52, 70-76]. Ongoing work in Canada [77] and Australia [78] strives to accurately track coverage levels, but an important companion to this work is a more detailed understanding of the barriers and supports to vaccination in Indigenous peoples. Moreover, generic interventions aimed at improving vaccine uptake in the general population of pre-adolescent children may require modifications or redesign for Indigenous peoples $[79,80]$, particularly given the multijurisdictional complexities in health services delivery for those living on reserve. In addition, context and pragmatics (e.g., differing HPV vaccination programs in municipal schools versus on-reserve schools) may impact coverage levels [81].

The evidence compiled thus far on the barriers, supports, and interventions for HPV vaccination reveals considerable gaps in data for Indigenous peoples. Even so, any evidence on vaccination in this population may provide important guidance for future work. To our knowledge, this will be the first synthesis of the barriers, supports, and interventions for improving vaccination and HPV vaccination in Indigenous peoples. Synthesislevel knowledge in this area is expected to support Indigenous peoples, researchers, clinicians, policy makers, and communities in their efforts to ameliorate welldocumented and disproportionate burdens of disease that persist in Indigenous populations worldwide.

\section{Methods/design}

\section{Aims}

Because we anticipate only a small number of relevant studies on HPV vaccination to emerge from the search, we will employ a broad search strategy to capture 
literature related to both HPV vaccination and vaccination in general among Indigenous peoples in Canada and globally. This systematic review will locate, gather, and critically appraise the global literature in Indigenous peoples [77], relating to (a) documented barriers and supports to vaccination and specifically $\mathrm{HPV}$ vaccination (V/HPV-V) and (b) interventions to increase acceptability and uptake or reduce hesitancy of $\mathrm{V} / \mathrm{HPV}-\mathrm{V}$.

The design of this mixed methods [82-84] systematic review protocol and search strategy was guided by the Cochrane Handbook for Systematic Reviews [85], the Preferred Reporting Items for Systematic Review and Meta-Analysis (PRISMA) [86] and protocols (PRISMA-P) [87], and ENTREQ [88] reporting guidelines. Because our study will generate both quantitative and qualitative data, we propose segregated analyses on each data set [89]; qualitative data will be managed using NVivo10 [90] and quantitative data will be captured and managed in MS Excel and analyzed using STATA v13.1 [91]. This study protocol was registered with the International Prospective Register of Systematic Reviews on March 1, 2017 (PROSPERO CRD42017048844). Any protocol amendments will be discussed as the review proceeds and documented using a protocol addendum in the final report [92, 93] (see Additional file 1).

\section{Research questions}

Primary questions of interest are:

1. What are the documented barriers and supports to $\mathrm{V} / \mathrm{HPV}-\mathrm{V}$, in Canadian Indigenous and global Indigenous peoples?

2. What are the documented interventions to increase acceptability and uptake or reduce hesitancy of $\mathrm{V} /$ $\mathrm{HPV}-\mathrm{V}$, in Canadian Indigenous and global Indigenous peoples?

Secondary questions of interest are:

3. What are the documented barriers and supports to $\mathrm{V} / \mathrm{HPV}-\mathrm{V}$, in youth (<18 years) within Canadian Indigenous and global Indigenous peoples?

4. What are the documented interventions to increase acceptability and uptake or reduce hesitancy of V/ HPV-V, in youth $(<18$ years) within Canadian Indigenous and global Indigenous peoples?

\section{Eligibility criteria}

We will include studies that involve global Indigenous peoples, including First Nations, Inuit, and Métis (Canada), American Indians and Alaskan Natives (USA), and Indigenous peoples in other countries including, but not limited to, China, South Asia, former Soviet Union,
Southeast Asia, South America, Africa, Central America/ Mexico, Arabia, Japan and the Pacific Islands, Australia, New Zealand, Greenland, and Scandinavia [69, 94, 95]. Study participants may include vaccine recipients, youth, parents, grandparents, guardians, and Elders and/or knowledge holders, health care providers, policy-makers, decision-makers, and community leaders, without restricting by sex or age. Draft inclusion-exclusion criteria were developed (Table 1) and refined using key articles identified from preliminary search findings. Eligible studies will (a) include global Indigenous peoples and (b) discuss barriers or supports and/or interventions to improve uptake/ acceptability or to reduce vaccine hesitancy for V/HPV-V. Studies will be excluded if they do not (a) include global Indigenous peoples or include subpopulation(s) of one or more global Indigenous peoples comprising more than $50 \%$ of study participants, or for which separate analyses for global Indigenous peoples or subpopulation(s) are not presented; (b) discuss barriers, supports or interventions for enhancing acceptability or uptake or reducing hesitancy for V/HPV-V; (c) report extractable data; or (d) report original research.

\section{Outcome measures}

Primary outcomes are documented barriers or supports or interventions that increase or enhance acceptability/ uptake or interventions to reduce hesitancy for V/HPVV. Secondary outcomes will investigate these same factors, specific to youth $(<18$ years) in Canadian and global Indigenous peoples. For this review, we define barriers as single or multilevel factors that are negatively associated with, or hinder the acceptability or uptake of $V / H P V-V$ or increase hesitancy. Supports include single or multilevel factors that are positively associated with, or enhance the acceptability and uptake of V/HPV-V or reduce hesitancy. Vaccination acceptability is defined as individuals' willingness to accept vaccination (typically measured by assessing attitudes, beliefs, and/or intention to be vaccinated) and vaccine uptake is defined as the behavior of getting vaccinated [40]. Vaccine hesitancy refers to a delay in acceptance or refusal of vaccines despite the availability of such services [41]. In order to optimize the capture of literature, vaccination intervention is defined as a singular or multilevel, simple and/or complex strategy(ies) used in the design or execution of vaccination or used to increase or enhance vaccine acceptability, uptake or to reduce vaccine hesitancy. Vaccination is defined as the act of introducing a vaccine into the body to produce immunity to a specific disease [96]. Study outcomes will be segregated into three main variable groups: study characteristics, barriers and supports, and vaccination interventions (acceptability, uptake, hesitancy). 
Table 1 Study inclusion and exclusion criteria

\begin{tabular}{l} 
Inclusion criteria \\
\hline Global Indigenous peoples (including First Nations, Inuit, and Métis \\
(Canada), American Indians and Alaskan Natives (USA), and Indigenous \\
peoples in other countries including, but not limited to, China, South \\
Asia, former Soviet Union, Southeast Asia, South America, Africa, Central \\
America/Mexico, Arabia, Japan and the Pacific Islands, Australia, New \\
Zealand, Greenland and Scandinavia) [69, 94] and participants may \\
include vaccine recipients, youth, parents, grandparents, guardians, \\
Elders and/or knowledge holders, health care providers, policy-/ \\
decision-makers, and community leaders, without restriction on gender \\
or age, \\
AND
\end{tabular}

Studies discuss barriers or supports to vaccination or HPV vaccination AND/OR

Studies discuss vaccination interventions to increase acceptability or uptake of vaccination or HPV vaccination, or to reduce vaccine or HPV vaccine hesitancy
Exclusion criteria

Studies that do not include global Indigenous peoples or in which one or more global Indigenous peoples comprise less than $50 \%$ of the study participants

OR

Studies that do not discuss barriers or supports to vaccination or HPV vaccination

AND/OR

Studies that do not discuss vaccination interventions, or HPV vaccination interventions or enhancing the acceptability or uptake of vaccination or HPV vaccination, or reducing vaccine or HPV vaccine hesitancy $\mathrm{OR}$

Studies lacking extractable data (i.e., policy papers or papers with no data)

$\mathrm{OR}$

Non-original research

\section{Study type}

All study designs meeting inclusion criteria will be considered, without restricting by language, location, or data type (i.e., qualitative, quantitative, and mixed methods studies are eligible).

\section{Data sources and search strategy}

A comprehensive search of the literature will use an a priori search strategy (draft MEDLINE search strategy in Table 1) comprised of key words and controlled vocabulary terms (Medical Subject Headings or MeSH terms). The core search strategy was developed in MEDLINE, in partnership with an academic librarian (HG) and submitted to a second academic librarian (YL) for independent peer review using the Peer Review of Electronic Search Strategies (PRESS) checklist [97]. PRESS feedback was integrated and the finalized strategy translated for use with the remaining databases. We will search several electronic databases (MEDLINE, CINAHL, EMBASE, Web of Science, PsycINFO, Global Index Medicus [LILACS and Western Pacific Region Index Medicus], Cochrane Database of Systematic Reviews, Joanna Briggs Institute Database, Scopus, Bibliography of Native North Americans, University of New Mexico Native Health Database, ProQuest Dissertations, Australian Indigenous HealthInfoNet) from first date of inception to September 2017, without restrictions (Table 2).

\section{Gray literature search strategy}

A search will be employed to identify original research that is not reported in peer-reviewed journal articles. The search and Level 1 screening process will be conducted concurrently, with two researchers searching a pre-defined group of government, agency, and Canadian and global Indigenous websites to locate relevant gray literature (Table 3). Combinations of the following combinations will be used: (1) Indigenous or Aboriginal or Indian, and (2) Vaccine or Vaccination or Immunization or Immunize or Immunization or Immunize or HPV or Human papillomavirus. Terms will be combined using "and" or " $\&$ ". Websites and resources that pertain to (1) Indigenous populations and (2) discuss barriers/supports or interventions to improve vaccination or HPV vaccination will be retained for screening. The title, URL, and data accessed for all websites/resources identified through this search will be entered into an Excel spreadsheet. Questionable resources will be retained for screening. All websites/resources identified in the search will be screened to determine if they meet the inclusion criteria (Table 1). Bibliographic searches will be conducted for all included studies and additional papers identified. We will also contact known provincial, national, and international experts working in this area (e.g., H. O’Donnell [Australia], S. Moore [Australia], R. Bednarczyk [United States]) for advice on relevant published and unpublished works.

\section{Preliminary findings}

A search of key national and international websites that register, fund, or publish knowledge syntheses failed to locate an existing synthesis to address our review questions. We identified several helpful systematic reviews in the areas of vaccine hesitancy [41, 60, 98], acceptability [60, 99], inequity of HPV vaccine uptake [100], barriers and facilitators to HPV vaccination in young women [25, 57, 61, 101, 102], and identification and assessment of interventions to improve HPV vaccine 
Table 2 Draft search strategy

Databases: Ovid MEDLINE(R) Epub Ahdead of Print, In-Process \& Other Non-Indexed Citations, Ovid MEDLINE(R) Daily and Ovid MEDLINE(R) 1946 to present (Feb 22, 2017)

The core search around the concepts of HPV and vaccination is as follows:

1. exp. Papillomavirus Infections/

2. exp. Vaccination/

3. (hpv or human papilloma* or vaccin* or immuniz*or immunis*).kw,tw.

4. 1 or 2 or 3

To identify studies involving Canadian indigenous populations, we applied a filter developed by health librarians at the University of Alberta [109] to identify Canadian indigenous populations to the above search.

We also ran this search against subject headings and keywords to identify studies related to selected global indigenous populations, as follows:

Databases: Ovid MEDLINE(R) Epub Ahead of Print, In-Process \& Other Non-Indexed Citations, Ovid MEDLINE(R) Daily and Ovid MEDLINE(R) 1946 to present (Feb 22, 2017)

1. exp. Papillomavirus Infections/

2. exp. Vaccination/

3. (hpv or human papilloma* or vaccin* or immuniz* or immunis*).kf,tw.

4. 1 or 2 or 3

5. exp. american native continental ancestry group/ or exp. oceanic ancestry group/

6. exp. Health Services, Indigenous/

7. exp. United States Indian Health Service/

8. (aborigin* or Alaska* Native* or American Indian* or Amerindian* or Eskimo* or community-based participatory or indigenous or Inuit* or Maori or Métis or Native American* or Native Hawaiian* or native people* or tribe* or tribal or Pacific Islander* or Torres Strait Islander*).kf,tw.

9. 5 or 6 or 7 or 8

10. 4 and 9

*denotes wildcard symbol in the search syntax. The wildcard broadens the search strategy by capturing the denoted word stem and all other derivatives beginning with the same letters

uptake $[64,79,80,103]$. We identified several tangential emergent syntheses through the PROSPERO International Prospective Register of Systematic Reviews pertaining to parental-reported barriers and facilitators to HPV vaccination [104], hesitancy toward HPV vaccine [105], the effects of mass media interventions on enhancing childhood vaccine uptake [106], health care provider recommendation of HPV vaccine in boys and young men $[107,108]$, and individual and environmentallevel factors influencing HPV vaccination [107]. A relevant review of specific subpopulations that included Canadian or global Indigenous peoples was not located. The core database search was conducted in MEDLINE using the Ovid interface, including in-process and e-pub-ahead-ofprint records, without restrictions, and generated a total of 2096 citations after de-duplication. A search filter for identifying literature for Canadian Indigenous peoples was added to the core MEDLINE search and feedback from PRESS review integrated [109].

\section{Study selection}

Search strategy findings will be cataloged using Endnote ${ }^{\mathrm{Tx}}$ X8.0 and duplicates removed manually. For inclusion, studies must involve one or more global Indigenous peoples and discuss barriers or supports to V/HPV-V and/or interventions to increase uptake/acceptability or reduce V/HPV-V hesitancy, as per study eligibility criteria. We will exclude studies that do not: a) involve a global Indigenous population (or a subpopulation comprising $\geq 50 \%$ of study participants), b) discuss barriers or supports to $\mathrm{V} /$ $\mathrm{HPV}-\mathrm{V}$; c) discuss $\mathrm{V} / \mathrm{HPV}-\mathrm{V}$ interventions or interventions that enhance the acceptability or uptake of V/HPV$\mathrm{V}$ or reduce $\mathrm{V} / \mathrm{HPV}-\mathrm{V}$ hesitancy, d) report extractable data, or e) report original research.

\section{Title and abstract screening (Level 1)}

Prior to commencing Level 1 (title and abstract) screening [89], we will calibrate screening among reviewers on a random sample (5\% of the total number of citations) [83]. All citations will then be screened independently and in duplicate by two investigators [GN/KJM/MSB/NB/SG/ $\mathrm{CC} / \mathrm{RVR}$ ] and discrepancies discussed until consensus is reached or, failing consensus, referred to a third reviewer for final resolution. Overall screening concordance will be calculated using the Kappa statistic [110]. Only those studies meeting screening inclusion/exclusion criteria will be retrieved for full text Level 2 [89] review. To pass Level 1 screening, studies must discuss one or more global indigenous peoples and barriers or supports to V/HPV-V or vaccination interventions to increase acceptability and uptake or reduce hesitancy for V/HPV-V.

\section{Full-text review and data abstraction (Level 2)}

The anticipated outcomes of interest are barriers and supports to V/HPV-V (qualitative and quantitative data, if measured) and interventions to enhance V/HPV-V acceptability or uptake or reduce hesitancy (qualitative and quantitative data, if measured), in Canadian and global Indigenous peoples and, secondarily, in Canadian Indigenous youth $(<18$ years). Where multiple citations occur, data will be combined to create a full description of the study. Should a lack of clarity about multiple citations arise, information will be sought from authors and studies excluded where discrepancies remain unresolved. We will calibrate full text screening among reviewers using the same methods described for Level 1 . The full text of each included citation will be reviewed independently and in duplicate by two investigators [CC/GN/ $\mathrm{KJM} / \mathrm{MSB} / \mathrm{NB} / \mathrm{SG} / \mathrm{SM} / \mathrm{RVR} / \mathrm{CB}$ ] and discrepancies resolved by a third investigator. 
Table 3 Gray literature - website search plan

\begin{tabular}{l}
\hline Gray literature resource name \\
Health Sciences Online (HSO) \\
OASter \\
International Network in Indigenous Health Knowledge and \\
Development (INIHKD) \\
Canadian Electronic Library's Public Documents Collection (previously \\
the Canadian Health Research Collection) \\
Center for World Indigenous Studies \\
Circumpolar Health Database \\
Health Info Net \\
Metis Health Database \\
Native Indigenous Studies Portal \\
Public Health Agency of Canada (PHAC)
\end{tabular}

First Nations Development Institute

Active Circle

Aboriginal Affairs and Northern Development (Government of Canada)

Centre for Indigenous Peoples' Nutrition and Environment (CINE)

First Nations Environmental Health Innovation Network

Assembly of First Nations

First Nations and Inuit Health Branch (Government of Canada - Health Canada)

National Collaborating Centre for Aboriginal Health (NCCAH) (Government of Canada)

National Aboriginal Health Organization (NAHO) (Agency was closed June 29, 2012; archival data will be searched using the Canadian Health Research Collection - archives available on the NAHO website to December 22, 2017)

Network Environment for Aboriginal Health Research (NEAHR)

BC Centres for Disease Control (Chee Mamuk - Aboriginal Health)

First Nations in BC Knowledge Network

First Nations Health Authority

Aboriginal Portal

Indigenous Affairs Website (Government of Alberta)

Ministry of Government Relations (First Nations Metis and Northern Affairs) (Government of Saskatchewan)

Centre for Aboriginal Health Research (CAHR)

First Nations, Metis and Inuit Health (University of Manitoba)

Indigenous and Municipal Relations (Government of Manitoba)

Ministry of Indigenous Relations and Reconciliation (Government of Ontario)

Centre for Indigenous Studies (University of Toronto)

Secretariat aux affaires autochtones (Gouvernement de Quebec)

Aboriginal Affairs (Government of New Brunswick)

Office of Aboriginal Affairs (Government of Nova Scotia)
URL

www.hso.info

http://oaister.worldcat.org/

http://www.inihkd.org

http://www.canadianelectroniclibrary.ca/Default.aspx

http://cwis.org/

http://www.aina.ucalgary.ca/chbd/

http://www.healthinfonet.ecu.edu.au/key-resources/bibliography

https://www.ccnsa-nccah.ca/en/

http://iportal.usask.ca/

http://www.phac-aspc.gc.ca/publicat/cdic-mcbc/index-eng.php? utm_source=subscription_list\&utm_medium=email_eng\&utm_ content=index\&utm_campaign $=C D \mid C \_32 \_2$

www.firstnations.org

www.activecircle.ca/en

https://www.aadnc-aandc.gc.ca/eng/1100100010002/ 1100100010021

https://www.mcgill.ca/cine/centre-indigenous-peoples-nutritionand-environment-cine

http://www.fnehin.ca

http://www.afn.ca/Assembly_of_First_Nations.htm

http://www.hc-sc.gc.ca/fniah-spnia/index-eng.php

http://www.nccah-ccnsa.ca/en/

http://www.naho.ca

http://cahr.uvic.ca/nearbc/elibrary/current-publications/

http://www.bccdc.ca/our-services/programs/chee-mamukaboriginal-health

http://fnbc.info

http://www.fnha.ca/

http://aboriginal.ubc.ca/

http://indigenous.alberta.ca

https://www.saskatchewan.ca/residents/first-nations-citizens/ saskatchewan-first-nations-metis-and-northern-affairs-directory

http://umanitoba.ca/centres/cahr/

http://umanitoba.ca/faculties/health_sciences/medicine/fnmi_ health/

https://www.gov.mb.ca/ana/

https://www.ontario.ca/page/ministry-indigenous-relations-andreconciliation

http://indigenousstudies.utoronto.ca

http://www.autochtones.gouv.qc.ca/index_en.asp

http://www2.gnb.ca/content/gnb/en/departments/aboriginal_ affairs.html

http://novascotia.ca/abor/office/ 
Table 3 Gray literature - website search plan (Continued)

Gray literature resource name
Aboriginal Affairs Secretariat (Government of Prince Edward Island)
Department of Labrador and Aboriginal Affairs Office (LAAO)
(Government of Newfoundland \& Labrador)
Aboriginal Relations (Government of Yukon)
NWT Aboriginal Governments

Aboriginal Affairs and Intergovermental Relations (Government of the North West Territories)

Department of Executive and Intergovermental Affairs (Aboriginal Affairs) Government of Nunavut

Population Research and Outcomes Studies Unit (PROS) (University of Melbourne)

Aboriginal and Torres Strait Islander-Australian Government Policy and Program Connection Website (Government of Australia - and State Site Portal)

Australian Institute of Health and Welfare

Australian Policy Online

The Lowitja Institute (Australia's National Institute for Aboriginal and Torres Strait Islander Health Research)

National Centre for Immunization Research and Surveillance (NCIRS)

(Government of Australia)

Immunize Australia

Toi Te Ora Public Health (Government of New Zealand)

New Zealand Ministry of Health

US Centers for Disease Contro

US Indian Health Service (Federal Health Program for American Indians and Alaska Natives)

Immunize Canada

Ministry of Health-BC

\section{BC Centre for Disease Control}

Immunize BC

Alberta Health

Alberta Health Services

Immunize Alberta

SK Ministry of Health

Manitoba Ministry of Health, Seniors and Active Living

Ontario Ministry of Health and long-term care

Public Health Ontario

Quebec Ministry of Health

Institut national de santé publique du Québec (INSPQ)

New Brunswick Ministry of Health

Nova Scotia Department of Health and Wellness

Nova Scotia Health Authority

PEI Health and Wellness

Health PEI

\section{URL}

http://www.gov.pe.ca/aboriginalaffairs/

http://www.laa.gov.nl.ca/laa/

http://www.eco.gov.yk.ca/aboriginalrelations/

http://www.daair.gov.nt.ca/en/nwt-aboriginal-governments

http://www.daair.gov.nt.ca

http://www.gov.nu.ca/eia/information/aboriginal-affairs

https://health.adelaide.edu.au/pros/

http://www.indigenous.gov.au/

http://www.aihw.gov.au/publication-detail/?id=10737419754

http://apo.org.au

http://www.lowitja.org.au

http://www.ncirs.edu.au/

http://www.immunise.health.gov.au/

https://www.govt.nz/organisations/toi-te-ora-public-healthservice/

https://www.health.govt.nz/

http://www.cdc.gov

https://www.ihs.gov

https://immunize.ca/

http://www2.gov.bc.ca/gov/content/governments/ organizational-structure/ministries-organizations/ministries/ health

http://www.bccdc.ca/

http://www.immunizebc.ca/

http://www.health.alberta.ca/

http://www.albertahealthservices.ca/

http://immunizealberta.ca/

https://www.saskatchewan.ca/government/governmentstructure/ministries/health

http://www.gov.mb.ca/health/

http://www.health.gov.on.ca/en/

http://www.publichealthontario.ca/en/Pages/default.aspx

http://www.msss.gouv.qc.ca/index.php

https://www.inspq.qc.ca/en

http://www2.gnb.ca/content/gnb/en/departments/health.html

https://novascotia.ca/DHW/

http://nshealth.ca/

https://www.princeedwardisland.ca/en/topic/health-and-wellness

http://www.healthpei.ca/

http://www.health.gov.nl.ca/health/ 
Table 3 Gray literature - website search plan (Continued)

\begin{tabular}{|c|c|}
\hline Gray literature resource name & URL \\
\hline \multicolumn{2}{|l|}{$\begin{array}{l}\text { Newfoundland and Labrador Department of Health and Community } \\
\text { Services }\end{array}$} \\
\hline $\begin{array}{l}\text { Canadian Association for Immunization Research and Evaluation } \\
\text { (CAIRE) }\end{array}$ & http://www.caire.ca/about/ \\
\hline Canadian Center for Vaccinology & http://centerforvaccinology.ca/ \\
\hline Vaccine Evaluation Center & http://vaccineevaluationcenter.ca/ \\
\hline Canadian Immunization Research Network (CIRN) & http://cirnetwork.ca/ \\
\hline Yukon Government Department of Health and Social Services & http://www.hss.gov.yk.ca/ \\
\hline NWT Health and Social Services & http://www.hss.gov.nt.ca/ \\
\hline Nunavut Department of Health & http://www.gov.nu.ca/health \\
\hline Canadian Public Health Association & http://www.cpha.ca/en/default.aspx \\
\hline Australian Indigenous Health Info Net & http://www.healthinfonet.ecu.edu.au/ \\
\hline
\end{tabular}

A draft data abstraction tool will be generated in MS Excel [111], based on the Data Collection Checklist from the Cochrane EPOC [112]. A subsection of the tool will be developed to assess the included intervention studies, specifically. We will use a set of a priori variables generated from preliminary findings (Table 4), combined with several items adapted from the Community Guide's Data Abstraction Framework (CGDA) [113] and the Standards for Reporting Implementation Studies Statement (StaRI) [114]. The abstraction tool will be pilot-tested by reviewers on a random sample of included articles and revised until consensus is reached on key variables and definitions.

\section{Methodological quality}

Methodological quality will be assessed using a universal appraisal tool (Quality Assessment Tool for Reviewing Studies with Diverse Designs - QATSDD) developed for the assessment of quality in diverse study designs [115]. The 4-point tool scoring system will be applied to each item, allowing an overall quality assessment for each study using one set of criteria. Team members will independently pilot the quality assessment tool using two to three included studies. The team will review discrepancies and discuss to consensus at a weekly team meeting before proceeding. Two investigators [CC/GN/KJM/ $\mathrm{MSB} / \mathrm{NB} / \mathrm{SG} / \mathrm{SM} / \mathrm{RVR} / \mathrm{CB}$ ] will assess methodological quality in all included studies, independently and in duplicate. Discrepancies will be noted and discussed until consensus, or failing consensus, referred to a third investigator. Quality scores will be reported as combined reviewer scores across the 16 items and the total score for each study and then summarized and used to fully describe the nature and characteristics of included studies in the analysis. Quality assessment scores will be presented in tabular format for all included studies to highlight the strengths and weaknesses of the evidence base.
Quality assessments will be used to 1) describe and characterize the methodological rigor and scientific quality of included studies; and 2) provide context for the interpretation of findings, formulation of conclusions, and/or recommendations arising from the review. Extreme outlier scores will be discussed by the team and depending on the issues identified, may be excluded to avoid the influence of significantly flawed studies on statistical and/or conceptual analyses, as appropriate.

A secondary quality analysis of the included effective intervention studies will be undertaken using the domains of the Consolidated Framework for Implementation Research (CFIR) [116]. The main purpose of this quality analysis is to gather and assess, where available, detail about the presence and nature of the individuallevel factors and contextual, intervention or implementation process factors for implementation, using a robust framework.

\section{Data analysis}

The overall study flow will be reported using a PRISMA diagram [86], providing a summary of citation numbers, sources, exclusions/rationale, and final number of included studies. Data analysis of abstracted variables will consist of two main sections with several subsections [82], including: (1) an inductive, qualitative, thematic synthesis [117] of all documented barriers and supports and (2) a quantitative analysis of measured barriers and supports found to be statistically significant, summarized as mean proportion and standard deviation of participants reporting the barrier or support (or other relevant aggregate measures as appropriate) or ranked by frequency of reported barriers or supports. The thematic analysis will proceed using a step-wise, iterative approach. Team members will review each study independently, and in duplicate. Line-by-line coding of identified barriers and/or supports will be conducted on 
Table 4 Level 2 (full text review) draft data abstraction tool (variables and definitions)

\begin{tabular}{|c|c|}
\hline Variable & Definition \\
\hline \multicolumn{2}{|l|}{ Study characteristics } \\
\hline Refid & Study unique reference identification number \\
\hline Author & Author last name, initials \\
\hline Year & Year of publication \\
\hline Title & Full title of manuscript \\
\hline Language & Original language of publication \\
\hline Location & Geographic location (country) of study \\
\hline Time & Data collection period (start to end of recruitment) \\
\hline Data & Data collection method(s) \\
\hline Funding & Reported funding source(s) for study \\
\hline Population & Indigenous population, community or ethnicity, as defined by study authors and eligibility criteria as reported by authors \\
\hline Target & Target population (health care providers, vaccine recipients, parents, policy makers etc.) \\
\hline Report(s) & Source(s) of reported barriers and/or supports \\
\hline Number & Number of participants in study \\
\hline age_mean, age_sd & Age of included participants [mean (SD), median (IQR), or categorical age, as reported] \\
\hline \multicolumn{2}{|l|}{ age_med, age-iqr } \\
\hline \multicolumn{2}{|l|}{ age_cat } \\
\hline Youth & Study participants (or cohort of study participants) were $<18$ years ( $Y e s=1$, No =0, Not Reported NR $=99$ ) \\
\hline Gender & $\%$ female \\
\hline Gender_target & Female, male, female and male \\
\hline Context & $\begin{array}{l}\text { Describe context of implementation (may include a description of the social, economic, policy, healthcare, organizational or } \\
\text { other contextual facets that may influence scale implementation) }\end{array}$ \\
\hline Setting & $\begin{array}{l}\text { Describe study setting, as defined by study authors (may include characteristics of the locations, personnel, resources for } \\
\text { implementation and/or criteria for eligibility as an implementation site that are used in the study }\end{array}$ \\
\hline setting_other & Describe 'other setting', as defined by study authors \\
\hline Aimobj & Study aims and objectives \\
\hline \multirow[t]{12}{*}{ Design } & Study design \\
\hline & $0=\mathrm{RCT}($ Randomized controlled trial) \\
\hline & $1=\mathrm{NRCT}$ (non-randomized controlled trial) \\
\hline & $2=$ cohort \\
\hline & $3=$ case control \\
\hline & $4=$ controlled before-after \\
\hline & $5=$ interrupted time series \\
\hline & $6=$ qualitative \\
\hline & $7=$ cross-sectional survey \\
\hline & $8=$ scoping, systematic or other synthesis \\
\hline & $9=$ mixed methods \\
\hline & $10=$ other \\
\hline design_other & Describe 'other study design' \\
\hline Sampling & Describe sampling strategy \\
\hline Inclusion & Study inclusion criteria, as reported by study authors \\
\hline Exclusion & Study exclusion criteria, as reported by study authors \\
\hline vaccine_delivery & Agency, authority, or group(s) administering vaccine \\
\hline vaccine_setting & The environment (clinic, school or other) where the vaccine was delivered \\
\hline vaccine_type, vaccine_batch & Type and/or batch of vaccine administered \\
\hline vaccine_series & Describe how the vaccine administered (one dose, 3 doses etc.) \\
\hline vaccine_status & Reported vaccination status of study participants (Yes $=1, \mathrm{No}=0$ ) \\
\hline
\end{tabular}


Table 4 Level 2 (full text review) draft data abstraction tool (variables and definitions) (Continued)

\begin{tabular}{|c|c|c|}
\hline Variable & \multicolumn{2}{|l|}{ Definition } \\
\hline vaccine_statusdescribe & \multicolumn{2}{|l|}{ If yes, describe whether full series, or only partial vaccination etc. } \\
\hline mod_frame & \multicolumn{2}{|c|}{ Authors describe explicitly, a health model, framework or theory utilized to frame their study (Yes $=1$, No =0, Not clear $=99$ ) } \\
\hline mod_frame_valid & \multicolumn{2}{|c|}{ Did the researchers use or test the model/framework or theory? (Yes $=1, \mathrm{No}=0$, Not reported $=99$ ) } \\
\hline mod_framedescribe & \multicolumn{2}{|l|}{ Describe model, framework or theory } \\
\hline Studylim & \multicolumn{2}{|l|}{ Reported study limitations } \\
\hline Onoffres & \multicolumn{2}{|l|}{ Location of participants (on reserve, off reserve) } \\
\hline onoffres_other & \multicolumn{2}{|c|}{ Describe location of participants if participants are located off reserve } \\
\hline Geography & \multicolumn{2}{|c|}{ Relative geographic location as reported by study authors } \\
\hline Ses & \multicolumn{2}{|l|}{ Reported socioeconomic status of participants } \\
\hline education_level & \multicolumn{2}{|l|}{ Reported level of education of participants } \\
\hline knowledge_gen* & \multicolumn{2}{|l|}{ Reported participant knowledge about vaccination } \\
\hline attitudes_gen* & \multicolumn{2}{|l|}{ Reported participant attitudes about vaccination } \\
\hline beliefs_gen* & \multicolumn{2}{|l|}{ Reported participant beliefs about vaccination } \\
\hline behaviors_gen* & \multicolumn{2}{|l|}{ Reported participant behaviors regarding vaccination } \\
\hline knowledge_HPV* & \multicolumn{2}{|l|}{ Reported participant knowledge about HPV vaccination } \\
\hline attitudes_HPV* & \multicolumn{2}{|l|}{ Reported participant attitudes about HPV vaccination } \\
\hline beliefs_HPV* & \multicolumn{2}{|l|}{ Reported participant beliefs about HPV vaccination } \\
\hline behaviors_HPV* & \multicolumn{2}{|l|}{ Reported participant behaviors regarding HPV vaccination } \\
\hline HPV_aware & \multicolumn{2}{|l|}{ Awareness of HPV vaccine } \\
\hline sex_status & \multicolumn{2}{|l|}{ Participants reporting sexually active } \\
\hline pap_status & \multicolumn{2}{|l|}{ Participants reporting prior abnormal pap smear test } \\
\hline STI_status & \multicolumn{2}{|l|}{ Participants reporting previous STI } \\
\hline HPV_status & \multicolumn{2}{|l|}{ Participants reporting prior HPV infection } \\
\hline Barriers and Supports & & \\
\hline barrier* $^{*}$ & Describe barriers to vaccine (all types) & subcategories will be determined inductively \\
\hline barrier_descr & Describe which vaccine type the reported barriers discuss & \\
\hline barrier_HPV* & Describe barriers to HPV vaccine & subcategories will be determined inductively \\
\hline support* & Describe supports to vaccine (all types) & subcategories will be determined inductively \\
\hline support_descr & Describe which vaccine type the reported supports discuss & \\
\hline support_HPV* & Describe supports to HPV vaccine & subcategories will be determined inductively \\
\hline recommend_gen* & Describe study recommendations for vaccination (all types) & subcategories will be determined inductively \\
\hline recommend_descr & Describe which vaccine type the recommendations pertain $t$ & \\
\hline recommend_HPV* & Describe study recommendations for HPV vaccination & subcategories will be determined inductively \\
\hline Vaccine Intervention Outc & & \\
\hline Outcomeimplem & Describe primary and other outcomes of the implementation & and how they were measured \\
\hline Outcomeintervent & Describe primary and other outcomes of the intervention, an & y were measured \\
\hline Process & Describe the process evaluation objectives and outcomes rel & mechanism by which the strategy is expected to work \\
\hline Intervention* & Describe vaccine intervention & subcategories will be determined inductively \\
\hline intervention_HPV* & Describe HPV vaccine intervention & subcategories will be determined inductively \\
\hline implemintervent & Describe the implementation strategy & \\
\hline HPVimplemintervent & Describe the HPV-specific implementation strategy & \\
\hline Subgroups & Describe any subgroups or nested studies undertaken in the & \\
\hline RR & Risk ratios reported & \\
\hline RR_pvalue & Reported $p$-values for RR & \\
\hline $\mathrm{OR}$ & Odds ratios reported & \\
\hline OR_pvalue & Reported $p$-values for OR & \\
\hline
\end{tabular}


Table 4 Level 2 (full text review) draft data abstraction tool (variables and definitions) (Continued)

\begin{tabular}{|c|c|}
\hline Variable & Definition \\
\hline Econintervent & Methods for resource use, costs, economic outcomes and analysis for the intervention \\
\hline Econimplem & Methods for resource use, costs, economic outcomes and analysis for the implementation strategy \\
\hline Samplesize & Describe the sample sizes, calculations \\
\hline Analysis & Describe analytic methods with rationale for methodological choice \\
\hline Subanalysis & Describe subanalyses, representativeness and outcomes of subgroups including those recruited to specific research tasks \\
\hline Fidelimplem & Fidelity of the implementation strategy as planned and adaptation to suit context and preferences \\
\hline Fidelintervent & Fidelity of delivering the core components of the intervention \\
\hline Contextchange & Describe contextual changes that may affect outcomes \\
\hline Harms & Describe all important harms or unintended effects for each group \\
\hline vaccine_acceptance & Reported factors influencing vaccine acceptance (study participant attitudes and beliefs) \\
\hline vaccine_uptake & A rate or quantification of the number or proportion of the study population who received vaccine intervention \\
\hline vaccine_refuse & A rate or quantification of the number or proportion of the population who refused vaccine intervention \\
\hline vaccine_hesitancy & $\begin{array}{l}\text { Describe reported factors relating to under-immunization, delay or questioning of vaccines, selecting only certain vaccines, } \\
\text { desire to access a trustworthy healthcare provider [Specific description of hesitancy as a barrier (all types of vaccine)] }\end{array}$ \\
\hline hesitancy_HPV & $\begin{array}{l}\text { Describe reported factors relating to under-immunization, delay or questioning of vaccines, selecting only certain vaccines, } \\
\text { desire to access a trustworthy healthcare provider [Specific description of hesitancy as a barrier (HPV vaccination)] }\end{array}$ \\
\hline CDGA_IC & CGDA Intervention Category [142] - Informational, Behavioral, Environmental \\
\hline CGDA_IL & $\begin{array}{l}\text { CDGA Intervention Intensity Level [142] - active engagement to individual, active engagement to population, passive } \\
\text { engagement with significant effort, passive engagement with minimal effort }\end{array}$ \\
\hline Interventimplic & Describe policy, practice or research implications for the intervention, including sustainability \\
\hline Imlemimplic & Describe policy, practice or research implications for the implementation strategy, specifically related to scalability \\
\hline Regulatory & Ethics approval, trial/study registration (and protocol if available), governance of data use, presence of conflicts of interest etc. \\
\hline
\end{tabular}

each study, and we will note similarities and differences in codes and discuss any discrepancies to consensus at weekly team meetings. Subsequent studies will be subjected to the same analysis, contributing text to existing (or generating new) themes, as appropriate. Once the coding process is complete, the team will review each of the barrier and support themes and sub themes, refine their definitions if necessary, and examine the text within them for consistency, code interrelations and to identify any potential conceptual hierarchies. All changes to codes, conceptual re-alignments, discussions and decisions will be documented as part of the study audit trail. We will report a summary of emerging themes, sub themes, definitions and linkages to studies in which themes arise.

We anticipate the ability to provide both a descriptive synthesis and quantitative summary of relevant measures for interventions; however, recent reviews examining barriers and supports in the area of HPV vaccination revealed a level of heterogeneity among studies precluding meta-analysis [25]. All included studies describing interventions will be assessed using the Community Guide's Data Abstraction Framework [118] with interventions categorized and frequencies reported by type and intensity.

We plan to report findings pertaining to Canadian Indigenous and global Indigenous peoples separately, contingent on the volume of eligible literature identified in each group. We will report results for HPV vaccines separately from results arising from studies of other vaccines, given the characteristics and contextual factors associated with HPV compared with other vaccines and target populations. A draft analysis plan and anticipated products of the synthesis are outlined in Table 5 .

\section{Discussion}

To date, we have not located a systematic review describing the barriers and supports to HPV vaccination or one that gathered and assessed effective interventions to increase acceptability and uptake of HPV vaccines in Indigenous peoples. Previous systematic reviews have been limited to vaccine efficacy in Indigenous peoples [119-121]. In addition to identifying studies that describe the barriers and supports to vaccination and interventions to increase vaccination acceptability and uptake in Indigenous peoples, the proposed systematic review will also aim to identify studies that specifically describe the barriers and supports to HPV vaccination and interventions that have been shown to increase acceptability and uptake of the HPV vaccine. While there is some evidence to suggest that tailoring vaccine strategies to the needs and preferences of adolescents using technology interventions may be promising, it is unclear how these findings relate to Canadian Indigenous youth. We have 


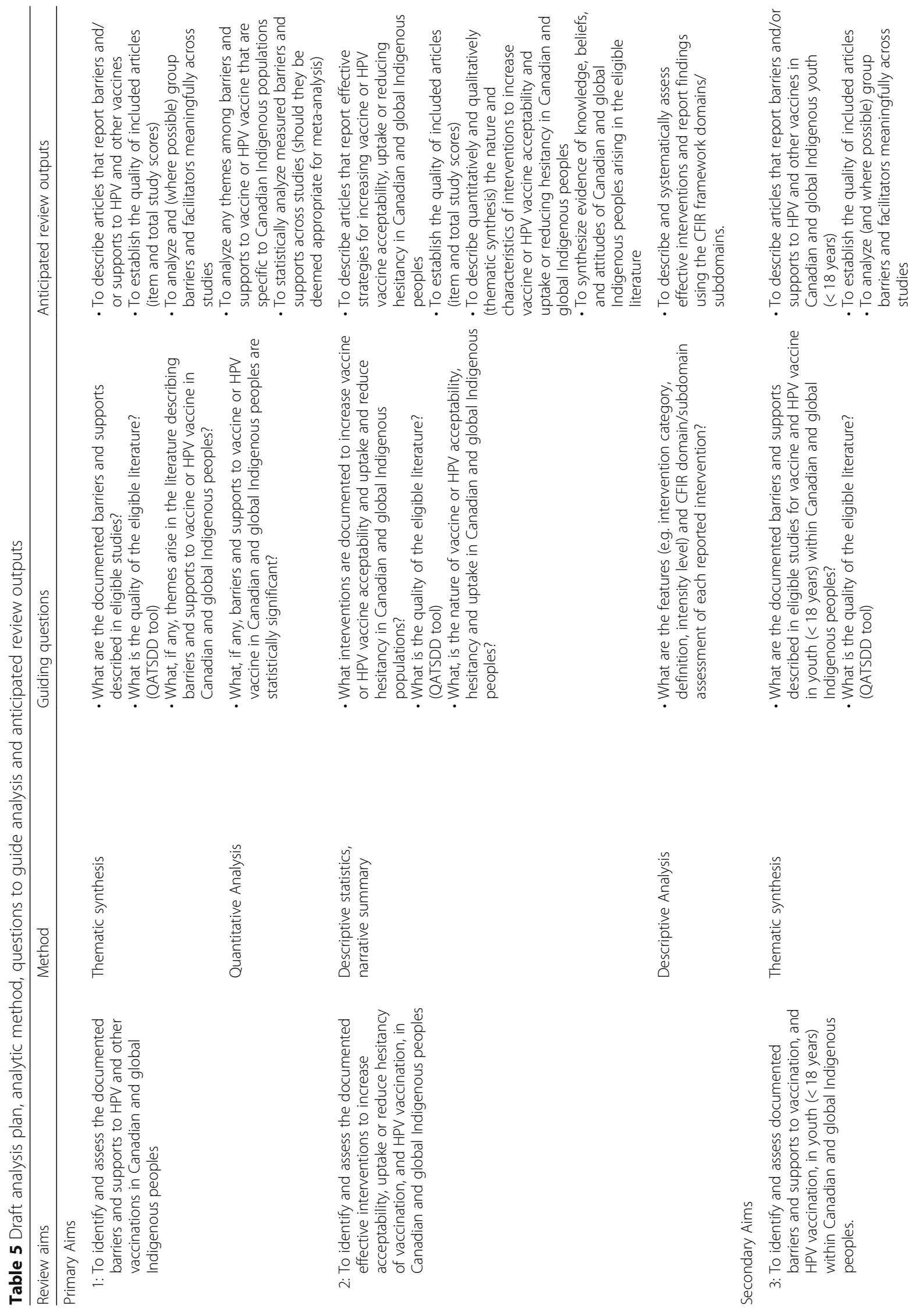




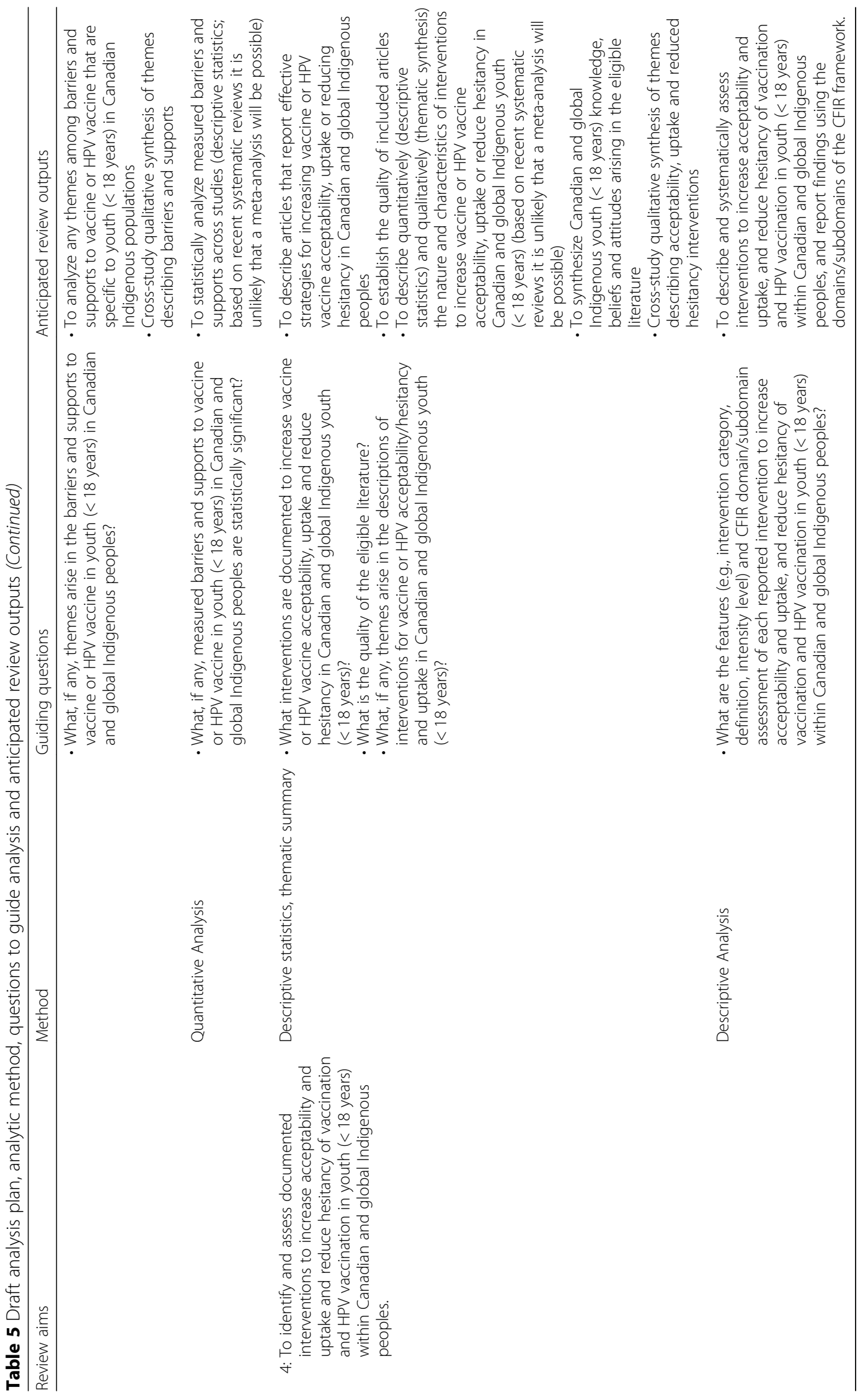


chosen to focus on HPV vaccination because, despite wide-spread availability, uptake of HPV vaccination between Indigenous and non-Indigenous youth remains unequal [19]. This finding is similar to that found in non-Indigenous populations in the USA where only $65 \%$ of girls and $56 \%$ of boys initiate a HPV vaccination series and fewer (43\% and $32 \%$, respectively) complete the series [64]. The approach we propose will allow us to synthesize existing evidence of HPV vaccine-specific barriers, supports, and effective interventions that may provide insights into vaccination practices that better meet the needs of Indigenous peoples.

While the importance of multiple $[79,80,122]$ and/or tailored [123, 124] (versus singular) interventions in some target groups remains a matter of debate in the implementation literature [125], multiple strategies focused on increasing HPV vaccination rates in non-Indigenous peoples have been shown to be effective [80, 126-132]. Furthermore, interventions tailored to address specific barriers to HPV vaccination or to address contextual differences may be required [133, 134]. It is unlikely, given the apparent state of the literature, that detailed information is available about contextual influences on HPV vaccination barriers and supports and HPV vaccination acceptability/hesitancy and uptake.

We have chosen to include global Indigenous peoples $[133,134]$ and anticipate that this review may suggest that Indigenous peoples, regardless of country, may experience similar barriers and supports to vaccination acceptability/hesitancy and uptake. With this in mind, we chose a broad definition of Indigenous peoples to help generate results that are relevant to many stakeholders. Given the dearth of literature in this area, we will consider all study designs meeting inclusion criteria, without restriction by language, location, or data type. We anticipate only a small number of relevant studies to emerge from the search and, hence, have used a broad, less restrictive search strategy that aims to capture literature related to V/HPV-V among Indigenous peoples.

The results of this study are expected to inform future research, policies, programs, and community-driven initiatives to enhance acceptability and uptake of vaccination among Indigenous peoples. Specifically, this review will identify and describe the documented barriers, supports, and interventions for V/HPV-V. These will provide a foundation to inform the development of new vaccination strategies among Indigenous peoples. We anticipate the review will identify the nature of evidence gaps and highlight areas requiring further study.

\section{Dissemination and knowledge translation plan}

To our knowledge, this study will contribute the first systematic review of the global literature in this area. We will take an integrated knowledge translation $[135,136]$ approach to guide our synthesis in close partnership with First Nation knowledge holders and others who will help interpret, structure, and disseminate findings to relevant research, clinical, Indigenous, policy, and decision maker groups. We anticipate there may be important regional, group, and contextual differences present in the data that will require tailored dissemination (EHVINA Study Research Team: Alberta First Nations Health Care Professional Stakeholder Meeting (2015), unpublished; EHVINA Study Research Team: EHVINA study - Alberta First Nations Elders Gathering (2015), unpublished; EHVINA Study Research Team: Canadian Institutes for Health Research - Institute for Cancer Research Stakeholder Engagement Brainstorming Session (2017), unpublished; EHVINA Study Research Team: Exploring International Collaborative Connections (2016), unpublished) and plan to co-present findings with First Nation partners. We anticipate strong receptivity for study findings and will utilize a translation process that is informed by the Knowledge-to-Action framework $[137,138]$ but is deliberately inductive to ensure knowledge systems, contexts, and the unique needs of our knowledge users are considered [77]. This process will extend from the analysis and interpretation of review findings through the conceptualization, mapping, and execution of vaccination strategies [139-141]. In closing, the intent of this synthesis is to assess the global literature pertaining to barriers and supports to V/HPV-V and identify effective interventions to enhance acceptability and uptake of V/HPV-V, in Canadian and global Indigenous peoples. We anticipate that this synthesis will provide numerous stakeholder groups with a better understanding of the current state of the literature in this area and help inform the development of interventions that encourage V/HPV-V acceptability and uptake and reduce hesitancy in Canadian and global Indigenous peoples.

\section{Additional file}

Additional file 1: PRISMA-P Checklist. (DOCX $39 \mathrm{~kb}$ )

\section{Abbreviations}

CDGA: Community guide's data abstraction framework (CGDA) (https:// www.thecommunityguide.org/about/our-methodology); CFIR: Consolidated framework for implementation research; ENTREQ: Enhancing transparency in reporting the synthesis of qualitative research: ENTREQ; EPOC: Cochrane effective practice and organization of care (epoc.cochrane.org/); FN: First nations; HPV: Human papilloma virus; KT: Knowledge translation; NVivo: qualitative data analysis software from QSR international (www.qsrinternational.com/nvivo/what-is-nvivo); PRESS: Peer review of electronic search strategies: 2015 guideline statement; PROSPERO: International prospective register of systematic reviews (https://www.crd.york.ac.uk/prospero/); QATSDD: Quality assessment tool for reviewing studies with diverse designs; STaRI: Standards for reporting implementation studies statement; STATA: Data analysis and statistical software program (https://www.stata.com/); URL: Universal resource locator; USA: United States of America; V/HPV-V: Vaccination and specifically HPV vaccination 


\section{Acknowledgements}

The team would like to thank Yongtao Lin for her involvement as a PRESS reviewer for the study search strategy, Simran Tiwana for her participation in early discussions of the protocol, and Markus Vaska for his assistance with the literature during grant development. We thank Caitlin Chalmers and Rhea Van Raay for their critical review of the protocol. This protocol is dedicated to the memory of Elder Mary Jane Wesley of Eden Valley, whose support of health prevention and promotion programs as an advisor and teacher to youth remain an enduring and inspirational memory at Eden Valley Health Centre, Alberta, Canada.

\section{Funding}

This work is funded by Alberta Innovates - Health Solutions through the Cancer Prevention Research Opportunity [2015 Collaborative Research and Innovation Opportunity (CRIO)] Grant \#201500852. The funding agency had no role in the conceptualization, design, or scripting of the systematic review protocol.

\section{Availability of data and materials}

Data sharing is not applicable to this article as no datasets were generated or analyzed during the current study.

\section{Authors' contributions}

KJM was involved with study conception and design, developed the protocol (search strategy, inclusion/exclusion criteria, quality assessment and data extraction), reviewed and approved the final manuscript. KJM also registered the protocol with PROSPERO. SM was involved with study conception and design, developed the protocol (search strategy, inclusion/ exclusion criteria, quality assessment and data extraction), reviewed and approved the final manuscript. MSB was involved with study conception and design, developed the protocol (search strategy, inclusion/exclusion criteria, quality assessment and data extraction), reviewed and approved the final manuscript. NB was involved with study conception and design, developed the protocol (search strategy, inclusion/exclusion criteria, quality assessment and data extraction), reviewed and approved the final manuscript. HG was involved with study conception and design, developed the protocol (search strategy, inclusion/exclusion criteria, quality assessment and data extraction), reviewed and approved the final manuscript. SG was involved with study conception and design, developed the protocol (search strategy, inclusion/ exclusion criteria, quality assessment and data extraction), reviewed, and approved the final manuscript. LB was involved with study conception and design, reviewed and approved the final manuscript. BH was involved with study conception and design, reviewed, and approved the final manuscript. $\mathrm{CH}$ was involved with study conception and design, reviewed, and approved the final manuscript. JG was involved with study conception and design, reviewed and approved the final manuscript. AC was involved with study conception and design, reviewed, and approved the final manuscript. CB was involved with study conception and design, reviewed and approved the final manuscript. RR was involved with study conception and design, reviewed and approved the final manuscript. $\mathrm{RH}$ was involved with study conception and design, reviewed and approved the final manuscript. JK was involved with study conception and design, reviewed and approved the final manuscript. CB was involved with study conception and design, reviewed, and approved the final manuscript. RAB was involved with study conception and design, reviewed, and approved the final manuscript. AL was involved with study conception and design, reviewed, and approved the final manuscript. GN was involved with study conception and design, developed the protocol (search strategy, inclusion/exclusion criteria, quality assessment and data extraction), reviewed, and approved the final manuscript. GN is the guarantor of the protocol. All authors read and approved the final manuscript.

\section{Authors' information}

KJM is a KT Implementation Scientist and Director (Knowledge Translation) with the Strategic Clinical Networks ${ }^{\text {tw }}$, Research Innovation and Analytics portfolio, Alberta Health Services and is a PhD trainee in the Department of Community Health Sciences, Cumming School of Medicine, University of Calgary. KJM is supported by an Alberta Health Services Post Secondary Education Tuition Award and Cumming School of Medicine Davies Scholarship for Academic Excellence, University of Calgary.
MSB is employed by the Arnie Charbonneau Cancer Institute, University of Calgary, and is an adjunct lecturer with the Department of Oncology, Cumming School of Medicine, University of Calgary.

GN is an Associate Professor, Department of Oncology and Obstetrics and Gynecology at the Cumming School of Medicine, University of Calgary, and as Tumor Group Leader, Gynecologic Oncology at the Tom Baker Cancer Centre, Alberta Health Services.

NB is a PhD trainee in the Department of Community Health Sciences, Cumming School of Medicine, University of Calgary and supported by an Alberta Innovates - Health Solutions Strategy for Patient Oriented Research Graduate Studentship (Joint Initiatives).

SM is an Assistant Professor in the Faculty of Nursing at the University of Alberta, and an Adjunct Assistant Professor in the Department of Pediatrics, Cumming School of Medicine, University of Calgary.

SG is an Assistant Professor, Department of Oncology and Obstetrics and Gynecology at the Cumming School of Medicine, University of Calgary. RAB is an Assistant Professor of Global Health and Epidemiology, Emory University Rollins School of Public Health, and a faculty member of the Emory Vaccine Center and the Winship Cancer Institute Cancer Prevention and Control Program.

AL is Science Lead, Community with the Alberta Cancer Prevention Legacy Fund, Population, Public and Aboriginal Health.

HG is an Associate Librarian, Head of Science \& Engineering (Gallagher Library), and Interim Head, Health Sciences Library, University of Calgary. RR is a Regional CDC Nurse Manager with Health Canada, First Nations and Inuit Health Branch, Alberta Region.

$\mathrm{CB}$ is an epidemiologist with the Analytics and Performance Reporting Branch, Alberta Health, Edmonton, Alberta.

CMB is an Associate Professor in the Departments of Medicine and Community Health Sciences, Cumming School of Medicine, University of Calgary. She is a ClHR New Investigator in Community Based Primary Health Care, and the Faculty Chair for the Group for Research with Indigenous Peoples (GRIP), in the O'Brien Institute for Public Health.

JK is Professor, Departments of Pediatrics, Microbiology, Immunology \& Infectious Diseases, and Community Health Sciences at the Cumming School of Medicine, University of Calgary.

LB is an Indigenous Baccalaureate-Prepared Registered Nurse and Indigenous Knowledge Holder at the Alberta First Nation Information Governance Centre.

$\mathrm{BH}$ is the Operations Manager of the Alberta First Nations Information Governance Centre.

$\mathrm{CH}$ is a Data Analyst with the Alberta First Nations Information Governance Centre.

AC is a Senior Epidemiologist with the Analytics and Performance Reporting Branch, Alberta Health.

JG is a Professor, Department of Community Health Sciences, University of Calgary.

$\mathrm{RH}$ is a Postdoctoral Fellow, Department of Community Health Sciences, University of Calgary.

Ethics approval and consent to participate Not applicable.

\section{Consent for publication}

Not applicable.

\section{Competing interests}

The authors declare that they have no competing interests.

\section{Publisher's Note}

Springer Nature remains neutral with regard to jurisdictional claims in published maps and institutional affiliations.

\footnotetext{
Author details

${ }^{1}$ Research Innovation and Analytics, Alberta Health Services, Edmonton, Canada. ${ }^{2}$ Department of Community Health Sciences, Cumming School of Medicine, University of Calgary, Calgary, Canada. ${ }^{3}$ Faculty of Nursing, University of Alberta, Edmonton, Canada. ${ }^{4}$ Arnie Charbonneau Cancer Institute, University of Calgary, Calgary, Canada. ${ }^{5}$ Department of Oncology, Cumming School of Medicine, University of Calgary, Calgary, Canada. ${ }^{6}$ Health Sciences Library, Libraries and Cultural Resources, University of Calgary,
} 
Calgary, Canada. ${ }^{7}$ Department of Obstetrics and Gynaecology, Cumming School of Medicine, University of Calgary, Calgary, Canada. ${ }^{8}$ Alberta First Nations Information Governance Centre, Calgary, Canada. ${ }^{9}$ Analytics and Performance Reporting Branch, Alberta Health, Edmonton, Canada. ${ }^{10} \mathrm{Health}$ Canada First Nations and Inuit Health Branch, Edmonton, Canada. ${ }^{11}$ Department of Paediatrics, Cumming School of Medicine, University of Calgary, Calgary, Canada. ${ }^{12}$ Hubert Department of Global Health, Rollins School of Public Health, Emory University, Atlanta, GA, USA. ${ }^{13}$ Alberta Cancer Prevention Legacy Fund, Edmonton, Canada.

\section{Received: 16 November 2017 Accepted: 22 January 2018} Published online: 02 March 2018

\section{References}

1. Munoz N, Bosch FX, de Sanjose S, Herrero R, Castellsague X, Shah KV, Snijders PJ, Meijer CJ. International agency for research on cancer multicenter cervical cancer study group: epidemiologic classification of human papillomavirus types associated with cervical cancer. N Engl J Med. 2003;348(6):518-27.

2. Jiang Y, Brassard P, Severini A, Mao Y, Li YA, Laroche J, Chatwood S, Corriveau A, Kandola K, Hanley B, Sobol I, Ar-Rushdi M, Johnson G, Lo J, Ratnam S, Wong T, Demers A, Jayaraman $\mathrm{G}$, Totten S, Morrison $\mathrm{H}$. The prevalence of human papillomavirus and its impact on cervical dysplasia in northern Canada. Infect Agents Cancer. 2013;8(25):1-11.

3. World Health Organization. Immunization, vaccines and biologicals: human papillomavirus (HPV). 201620 March 2016 [cited 2016 October 7].

4. Young TK, Kliewer E, Blanchard J, Mayer T. Monitoring disease burden and preventive behaviour with data linkage: cervical cancer among aboriginal people in Manitoba, Canada. Am J Public Health. 2000;90(9):1466-8.

5. Kjaer SK, Nielsen NH. Cancer of the female genital tract in circumpolar Inuit. Acta Oncol. 1996;35(5):581-7.

6. Louchini $\mathrm{R}$, Beaupre M. Cancer incidence and mortality among aboriginal people living on reserves and northern villages in Quebec, 1988-2004. Int J Circumpolar Health. 2008;67(5):445-51.

7. NWT Bureau of Statistics, Annual population estimates by community and other characteristics of the Northwest Territories, N.B.o. Statistics, Editor. 2009.

8. Public Health Agency of Canada., Cervical Cancer Screening in Canada: 1998 Surveillance Report, P.H.A.o.C. (PHAC), Editor. 1998.

9. Circumpolar Inuit Cancer Review Working Group. Cancer among the circumpolar Inuit, 1989-2003 II. Patterns and trends circumpolar Inuit. Int J Circumpolar Health. 2008;67(5):408-20.

10. Hamlin-Douglas LK, Coutlee F, Roger M, Franco EL, Brassard P. Prevalence and age distribution of human papillomavirus infection in a population of Inuit women in Nunavik, Quebec. Cancer Epidemiol Biomarkers Prev. 2008;17(11):3141-9.

11. Jiang $Y$, Brassard $P$, Severini A, et al. Type-specific prevalence of Humpan Papillomavirus infection among women in the northwest territories, Canada. J Infect Public Health. 2011;4(5-6):219-27.

12. Bennett $\mathrm{R}$, Cerigo $\mathrm{H}$, Coutlee $\mathrm{F}$, et al. Incidence, persistence and determinants of human papillomavirus infection in a population of Inuit women in northern Quebec. Sex Transm Dis. 2015;42(5):272-8.

13. Northwest Territories Health and Social Service. Cancer in the northwest territories 1990-2000: a descriptive report. Yellowknife: NWT; 2003.

14. McDermott S. Cervical cancer screening in the NWT. Epidemiol North. 2002;14:14-8.

15. Decker KM, Demers AA, et al. Pap test use and cervical cancer incidence in first nations women living in Manitoba. Cancer Prev Res. 2015;8(1):49-55.

16. Canada H. A statistical profile on the health of first nations in Canada. In: Health services utilization in western Canada, 2000-2009; 2000.

17. Nishri ED, Sheppard AJ, Withrow DR, et al. Cancer survival among first nations people of Ontario, Canada (1968-2007). Int J Cancer. 2015;136(3):639-45.

18. Sobol, I., Totten, S., et al.,. Burden of HPV disease in Nunavut. 2010; Available from: http://www.slideshare.net/NAHONews/sobol-hpv2010-july5nologo? next_slideshow $=1$.

19. Shafer LA, Jeffrey I, Elias B, Shearer B, Canfell K, Kliewer E. Quantifying the impact of dissimilar HPV vaccination uptake among Manitoban school girls by ethnicity using a transmission dynamic model. Vaccine. 2013;31(42):4848-55.

20. Society of Obstetricians and Gynecologists of Canada. Canadian consensus guidelines on human Papillomavirus. 2000 July 30, 2015]; Available from: http://sogc.org/wp-content/uploads/2013/01/gui196CPG0708revised_000.pdf.
21. Office of the Auditor General of Canada, Reports of the Auditor General of Canada. Access to health Services for Remote First Nations Communities. Ottawa, Canada: The Office of the Auditor General of Canada; 2015.

22. Pace $D$, Konczi AE. Alberta first nations information governance Centre. In: First nations regional health survey 2008-2010., in Alberta report, vol. 2012.

23. Kliewer E, Elias B, Demers A, Lambert P, Hall M. Uptake of the Human Papillomavirus Vaccine Among Manitoba's Status First Nations Females, 2006-2009. Manitoba: Cancer Care Manitoba; 2012. p. 1-23.

24. Joura EA, Giuliano AR, Iversen OE, et al. Broad Spectrum HPV vaccine study. A 9-valent HPV vaccine against infection and intraepithelial neoplasia in women. N Engl J Med. 2015;372(8):711-23.

25. Rambout L, Tashkandi M, Hopkins L, Tricco A. Self-reported barriers and facilitators to preventive human papillomavirus vaccination among adolescent girls and young women: a systematic review. Prev Med. 2014;58

26. Liu X, Bell C, Simmonds K, Svenson L, Russell M. Adverse events following HPV vaccination, Alberta 2006-2014. Vaccine. 2016;34(15):1800-5.

27. Government of Canada, Canadian Immunization Guide. Part 1 - key immunization information, vol. 1-10. Government of Canada, Ottawa, Canada; 2016.

28. Naus M. What do we know about how to improve vaccine uptake? Can Commun Dis Rep. 2015;41(S-3):1-5.

29. Public Health Agency of Canada. Immunization: the most successful public health measure. 2009 February 17, 2009 September 10, 2016]; Available from: http://www.phac-aspc.gc.ca/im/measure-intervention-eng.php.

30. Larson, H., \& Schulz, W., and the Vaccine Confidence Project Team,. The state of vaccine confidence 2015. 2015, London School of Hygiene \& Tropical Medicine: London, UK

31. Miller MA, Hinman AR. In: Plotkin SA, Orenstein WA, editors. Chapter 57 economic analyses of vaccine policies., in vaccines. Philadelphia, PA: Elsevier Inc.; 2004

32. Hadler SC. In: Plotkin SA, Orenstein WA, editors. Chapter 55 - vaccination programs in developing countries, in vaccines. Philadelphia, PA: Elsevier Inc.; 2004.

33. World Health Organization. A.U.N.C.S.F., global immunization vision and strategy, 2006-2015. Geneva, Switzerland: World Health Organization and United Nations Children's Fund; 2005.

34. Andre FE, Booy R, Bock HL, Clemens J, Datta SK, John TJ, Lee BW, Lolekha S, Peltola H, Ruff TA, Santosham M, Schmitt HJ. Vaccination greatly reduces disease, disability, death and inequity worldwide. Bull World Health Organ. 2007;86(2):1-13.

35. Colgrove J. Immunity for the people: the challenge of achieving high vaccine coverage in American history. Public Health Chronicles. 2007; 122(March-April):248-58.

36. World Health Organization. Immunization, vaccines and Biologicals: global immunization coverage sustained in the past five years. 2016 September 10 2016]; Available from: http://www.who.int/immunization/newsroom/press/ immunization_coverage_july_2016/en/.

37. World Health Organization. The guide to tailoring immunization Programmes (TIP): increasing coverage of infant and child vaccination in the WHO Europea region: World Health Organization - Regional Office for Europe, Copenhagen, Denmark; 2013. p. 1-79.

38. World Health Organization. Immunization, vaccines and Biologicals: immunization coverage. 2016 September 10, 2016]; Available from: http://www.who.int/ immunization/monitoring_surveillance/routine/coverage/en/.

39. Centers for Disease Control and Prevention. Vaccination coverage in the $U$. S. 2016 February 17, 2016 September 10, 2016]; Available from: http://www. cdc.gov/vaccines/imz-managers/coverage/imz-coverage.html.

40. World Health Organization, Report of the SAGE working group on vaccine hesitancy. 2014

41. World Health Organization SAGE Working Group Dealing with Vaccine Hesitancy, Strategies for Addressing Vaccine Hesitancy - A systematic review. Geneva: World Health Organization; 2014. p. 1-253.

42. Butler, R., MacDonald, N., and the SAGE Working Group on Vaccine Hesitancy., Diagnosing the determinants of vaccine hesitancy in specific subgroups: the guide to tailoring immunization programs (TIP). Vaccine, 2015. 33: p. 1-4

43. Falagas ME, Zarkadoulia E. Factors associated with suboptimal compliance to vaccinations in children in developed countries: a systematic review. Curr Med Res Opin. 2008;24:1719-41.

44. Dube E, Laberge C, Guay M, Bramadat P, Roy R, Bettinger J. Vaccine hesitancy an overview. Hum Vaccin Immunother. 2013;9(8):1763-73. 
45. Larson HJ, Jarrett C, Exkersberger E, Smith DM, Paterson P. Understanding vaccine hesitancy around vaccines and vaccination from a global perspective: a systematic review of published literature, 2007-2012. Vaccine. 2014;32(19):2150-9.

46. Brown K, Kroll J, Hudson M, Ramsay M, Green J, Long S, Sevdalis N. Factors underlying parental decisions about combination childhood vaccinations including MMR: a systematic review. Vaccine. 2010;28(26):4235-48.

47. Zhang J, Ohinmaa A, Nguyen T, Mashinter L, Hanrahan A, Loewen J, Jacobs PD. Determinants for immunization coverage by age 2 in a population cohort in the Capital Health region, Edmonton, Alberta. Can Commun Dis Rep. 2008;34(9):1-10.

48. Avis K, Tan L, Anderson C, Tan B, Muhajarine N. Taking a closer look: an examination of measles, mumps, and rubella immunization uptake in Saskatoon. Can J Public Health. 2007;98:417-21.

49. MacDonald NE. Vaccine hesitancy: definition, scope and determinants. Vaccine. 2015;33(34):4161-4

50. Mills E, Jadad AR, Ross C, Wilson K. Systematic review of qualitative studies exploring parental beliefs and attitudes toward childhood vaccination identifies common barriers to vaccination. J Clin Epidemiol. 2005;58(11):1081-8

51. Salmon DA, Moulton LH, Omer SB, DeHart MP, Stokley S, Halsey NA. Factors associated with refusal of childhood vaccines among parents of school-aged children: a case-control study. Arch Pediatr Adolesc Med. 2005;159(5):470-6.

52. MacDonald SE, Bell CA, Simmonds KA. Coverage and determinants of uptake for privately funded rotavirus vaccine in a Canadian birth cohort, 2008-2013. Pediatr Infect Dis J. 2016;35(6):e177-9.

53. Pearce A, Marshall H, Bedford H, Lynch J. Barriers to childhood immunization: findings from a longitudinal study of Australian children. Vaccine. 2015;33(29):3377-83.

54. Public Health Agency of Canada, An Advisory Committee Statement (ACS) National Advisory Committee on Immunization (NACl): Updated Recommendations on Human Papillomavirus (HPV) Vaccines: 9-valent HPV Vaccine and Clarification of Minimum Intervals Between Doses in the HPV Immunization Schedule., Public Health Agency of Canada, Editor. 2016, Her Majesty the Queen in Right of Canada, as represented by the Minister of Health: Ottawa, Ontario Canada. p. 1-56.

55. de Martel C, Ferlay J, Franceschi S, et al. Global burden of cancers attributable to infections in 2008: a review and synthetic analysis. Lancet Oncol. 2012;13:607-15.

56. Massad LS, Einstein M, Myers E, et al. The impact of human papillomavirus vaccination on cervical cancer prevention efforts. Gynecol Oncol. 2009;114(2):360-4.

57. Holman DM, Benard V, Roland KB, Watson M, Liddon N, Stokley S. Barriers to human Papillomavirus vaccination among US adolescents: a systematic review of the literature. JAMA Pediatr. 2014;168(1):76-82.

58. Hendry M, Lewis $R$, Clements A, Damery S, Wilkinson C. "HPV? Never heard of it!": a systematic review of girls' and parents' information needs, views and preferences about human papillomavirus vaccination. Vaccine. 2013;31:5152-67

59. Kessels SJM, Marshall HS, Watson M, Braunack-Mayer AJ, Reuzel R, Tooher $\mathrm{RL}$. Factors associated wtih PHV vaccine uptake in teenage girls: a systematic review. Vaccine. 2012;30(24):3546-56

60. Brewer NT, Fazekas KI. Predictors of HPV vaccine acceptability: a theoryinformed systematic review. Prev Med. 2007:45:107-14

61. Batista Ferrer $\mathrm{H}$, Trotter C, Hickman M, Audrey S. Barriers and facilitators to HPV vaccination of young women in high-income countries: a qualitative sytematic review and evidence synthesis. BMC Public Health. 2014;14(700):22.

62. Robbins SCC, Ward K, Skinner SR. School-based vaccination: a systematic review of process evaluations. Vaccine. 2011;29(9588-9599)

63. French SD, Green SE, O'Connor DA, McKenzie JE, Francis JJ, Michie S, Buchbinder R, Schattner P, Spike N, Grimshaw JM. Developing theoryinformed behaviour change interventions to implement evidence into practice: a systematic approach using the theoretical domains framework. Implement Sci. 2012;7(38):1-8.

64. Walling EB, Benzoni N, Dornfeld J, Bhandari R, Sisk BA, Garbutt J, Colditz G. Interventions to improve HPV vaccine uptake: a systematic review. Pediatrics. 2016;138(1)

65. Abuelo CE, Levinson KL, Slameron J, Sologuren CV, Fernandez MJV, Belinson JL. The Peru cervical cancer screening study (PERCAPS): the design and implementation of a mother/daughter screen, treat, and vaccinate program in the Peruvian jungle. J Community Health. 2014;39(3):409-15
66. Demers B, Shearer B, Totten S, et al. Prevalence of HPV infections in Metis and first nations living in Manitoba. Sex Transm Infect. 2011;87:A152.

67. Whop LJ, Garvey G, Baade P, Cunningham J, Lokuge K, Brotherton JM, et al. The first comprehensive report on indigenous Australian women's inequalities in cervical screening: a retrospective registry cohort study in Queensland, Australia (2000-2011). Cancer. 2016;122(10):1560-9.

68. Moore SP, Antoni S, Colquhoun A, Healy B, Ellison-Loschmann L, Potter JD, et al. Cancer incidence in indigenous people in Australia, New Zealand, Canada and the USA: a ocmparative population-based study. Lancet Oncol. 2015;16(15):1483-92.

69. IWGIA. 9th annual IWGIA 2008 report definition in: enhancing HPV prevention among indigenous populations: international perspectives on health. In: A symposium of the 26th international Papillomavirus conference, vol. 1. Montreal, Canada: George Wurtak International Centre for Infectious Diseases; 2010. p. 114

70. Hill HA, Elam-Evans LD, Yankey D, Singleton JA, Dietz V. Vaccination coverage among children aged 19-35 months - United States 2015. Morb Mortal Wkly Rep. 2016;65(39):1065-71.

71. Strine TW, Mokdad AH, Barker LE, Groom AV, Singleton R, Wilkins CS, Chu SY. Vaccination coverage of American Indian/Alaska native children aged 19-35 months: findings from the National Immunization Survey, 1998-2000. Am J Public Health. 2003;93(12):2046-9.

72. Lovie-Toon JG, Hall KK, Chang AB, Anderson J, O'Grady KF. Immunisation timelines in a cohort of urban aboriginal and Torres Strait islander children. BMC Public Health. 2016;16(1159)

73. Lemstra M, Neudorf C, Opondo J, Kurji A, Kunst A, Tournier C. Disparity in childhood immunizations. Pediatr Child Health. 2007;12(10):847-52.

74. Quach S, Hamid JS, Pereira JA, Heidebrecht CL, Deeks SL, Crowcroft NS, Quan SD, Brien S, Kwong JC. Influenza vaccination coverage across ethnic groups in Canada. Can Med Assoc J. 2012;184(15):1673-81.

75. National Centre for Immunization Research. Vaccine preventable diseases and immunization coverage in aboriginal and Torres Strait islander people, Australia 2006-2010. 2013; Available from: http://www.health.gov.au/ internet/publications/publishing.nsf/Content/cda-cdi37suppl.htm.

76. United Nations International Children's Emergency Fund (UNICEF). Canadian supplement to the state of the world's children 2009. In: Aboriginal Children's health: leaving no child behind. Toronto, Canada.: UNICEF Canada; 2009.

77. Nelson GS, Letendre A, Shea-Budgell MA, Tiwana S, Glaze S, Mrklas KJ, Guichon J, Kellner J, MacDonald S, Healy B, Kromm S, Bell C, Richardson R, Henderson R. Enhancing HPV vaccination in first nations populations in Alberta (EHVINA study): towards a sustainable, community-driven knowledge translation strategy [collaborative research and innovation opportunities (CRIO): cancer prevention research opportunity]. In: Alberta Innovates - Health Solutions (2015 CPRO-CRIO level 1 competition): Alberta, Canada; 2015. p. 1-25.

78. Brotherton JM, Murray SL, Hall MA, Andrewartha LK, Banks CA, Meijr D, et al. Human papillomavirus vaccine coverage among female Australian adolescents: success of the school-based approach. Med J Aust. 2013;199(9):614-7.

79. Fu LY, Bonhomme LA, Cooper SC, Joseph JG, Zimet GD. Educational interventions to increase HPV vaccination acceptance: a systematic review. Vaccine. 2014;32(17):1901-20.

80. Niccolai LM, Hansen CE. Practice- and community based interventions to increase human papillomavirus vaccine coverage: a systematic review. JAMA Pediatr. 2015;169(7):686-92.

81. Government of Canada, Regional communicable disease control report, 2016, F.N.a.I.H.B.-A.R.I. Report), Editor. 2016, Government of Canada: Ottawa, Ontario Canada.

82. Pluye P, Hong QN. Combining the power of stories and the power of numbers: mixed methods research and mixed studies reviews. Annu Rev Public Health. 2014;35:29-45.

83. Kastner M, Tricco AC, Soobiah C, Lillie E, Perrier L, Horsley T, Welch V, Cogo E, Antony J, Straus SE. What is the most appropriate knowledge synthesis method to conduct a review? Protocol for a scoping review. BMC Med Res Methodol. 2012:12(114):1-10.

84. Kastner M, Antony J, Soobiah C, Straus SE, Tricco AC. Conceptual recommendations for selecting the most appropriate knowledge synthesis method to answer research questions related to complex evidence. J Clin Epidemiol. 2016;73:43-9.

85. Higgins, J.P., \& Green, S. Cochrane handbook for systematic reviews of interventions. 2011 March 2011 [cited 2015 November 5]; Available from: http://handbook.cochrane.org. 
86. Moher D, Liberati A, Tetzlaff J, Altman D, PRISMA Group. Preferred reporting items for systematic reviews and meta-analyses: the PRISMA statement. Ann Intern Med. 2009;151(4):7.

87. Moher D, Shamseer L, Clarke M, Ghersi D, Liberati A, Petticrew M, Shekelle $P$, Stewart LA, PRISMA-P Group. Preferred reporting items for systematic review and meta-analysis protocols (PRISMA-P) 2015 statement. Syst Rev. 2015;4(1):1-9.

88. Tong A, Flemming K, McInnes E, Oliver S, Craig J. Enhancing transparency in reporting the synthesis of qualitative research: ENTREQ. BMC Med Res Methodol. 2012;12(181)

89. Tricco AC, Antony J, Soobiah C, Kastner M, MacDonald H, Cogo E, Lillie E, Tran J, Straus SE. Knowledge synthesis methods for integrating qualitative and quantitative data: a scoping review reveals poor operationalization of the methodological steps. J Clin Epidemiol. 2016;73:29-35.

90. International Q. NVivo10 for windows. New York, USA: QSR International; 2014.

91. StataCorp LP. STATA v13.1 special edition. USA: StataCorp LP: College Station; 2013.

92. Silagy CA, Middleton P, Hopewell S. Publishing protocols of systematic reviews: comparing what was done to what was planned. JAMA. 2002;287:2831-4.

93. Centre for Reviews and Dissemination (CRD), Systematic Reviews: CRD's guidance for undertaking reviews in health care., C.f.R.a. Dissemination, Editor. 2009, University of York: York, UK.

94. Hall, G., Indigenous peoples, poverty and development. Draft Manuscript seek full text In IPV_Final_Report_Jul52010__final_Jan 2011.pdf, 2010.

95. United Nations Permanent Forum on Indigenous Issues. "About UNPFII and a Brief History of Indigenous Peoples and the International System". 2006 September 10, 2016; Available from: https://www.un.org/development/ desa/indigenouspeoples/unpfii-sessions-2.html .

96. Centers for Disease Control and Prevention. Vaccines and immunizations: the basics - definition of terms. 2017 [cited 2017 Accessed on September 17, 2017]; Available from: https://www.cdc.gov/vaccines/vac-gen/imz-basics.htm.

97. Sampson M, McGowan J, Cogo E, Grimshaw J, Moher D, Lefebvre C. An evidence-based practice guideline for the peer review of electronic search straetgies. J Clin Epidemiol. 2009;62:944-52.

98. Dube E, Gagnon D, MacDonald NE. The SAGE working group on vaccine hesitancy, strategies intended to address vaccine hesitancy: review of published reviews. Vaccine. 2015;33:4191-203.

99. Newman PA, Logie CH, Doukas N, Asakura K. HPV vaccine acceptability among men: a systematic review and meta-analysis. Sex Transm Infect. 2013;89:568-74

100. Fisher H, Trotter C, Audrey S, Macdonald-Wallis K, Hickman M. Inequalities in the uptake of human papillomavirus vaccination: a systemtic review and meta-analysis. Int J Epidemiol. 2013;25(25)

101. Batista Ferrer H, Audrey S, Trotter C, Hickman M. An appraisal of theoretical approaches to examining behaviours in relation to human Papillomavirus (HPV) vaccination of young women. Prev Med. 2015;81:122-31.

102. Marshall S, Sahm L, Fleming A, Moore A. Knowledge, attitudes and bliefs regarding the adolescent human papillomavirus (HPV) vaccination: a systematic review and meta-ethnographic synthesis of the qualitative literature. In: PROSPERO international prospective register of systematic reviews; 2016.

103. Sadaf A, Richards JL, Glanz J, Salmon DA, Omer SB. A systematic review of interventions for reducing parental vaccine refusal and vaccine hesitancy. Vaccine. 2013;31:4293-304.

104. Ayino K. Vaccination for my daughter, maybe but no thanks: a research synthesis on parental explained barriers to delayed or non-acceptance of HPV vaccination in high income (OECD) nations. In: PROSPERO international prospective register of systematic reviews; 2016.

105. Khan T, Wong C, Yap Y. Vaccine hesitancy towards human Papillomavirus (HPV) vaccination in South East Asia region (SEAR) and western Pacific region (WPR): a systematic review. In: PROSPERO international prospective register of systematic reviews; 2016

106. Nguweneza A, Machingaidze S, Wiysonge CS, Zani B. A systematic review of the effects of mass media interventions to improev uptake of childhood vaccines. In: PROSPERO international prospective register of systematic reviews; 2015.

107. Rodriguez S, vonVille H. Individual and environmental-level factors influencing HPV vaccination: building a conceptual model through a systematic review of reviews. In: PROSPERO international prospective register of systematic reviews; 2016.

108. Newman PA, Logie CH, Rubincam C, Lacombe-Duncan A, Baiden P, Asey AF. Health care provider recommendation of HPV vaccines for boys and young men: a systematic review. In: PROSPERO international prospective register of systematic reviews; 2015.

109. Campbell, S., Dorgan, M., Tjosvold, L. Filter to Retrieve Studies Related to Indigenous People of Canada the OVID Medline Database. 2016 March 8, 2016 May 10, 2017]; Available from: http://guides.library.ualberta.ca/ld. php?content_id=20227853.

110. Altman DG. Practical statistics for medical research: measuring agreement. London, UK: Chapman and Hall; 1991.

111. Microsoft Corporation. Microsoft Excel for Mac. Redmond: Microsoft Corporation; 2010.

112. Cochrane Effective Practice and Organisation of Care (EPOC). Data collection form. EPOC resources for review authors 2017 [cited 2017 Accessed September 17, 2017]; Available from: http://epoc.cochrane.org/epocspecific-resources-review-authors.

113. Briss PA, Zaza S, Pappaioanou M, Feilding J, Wright-De Aguero L, Truman B, Hopkins D, et al. Developing an evidence-based guide to community preventive services - methods. Am J Prev Med. 2000;18(1):35-43.

114. Pinnock, H., Barwick, M., Carpenter, C.R., Eldridge, E., Grandes, G., Griffiths, C.J. , Roycroft-Malone, J., Meissner, P., Murray, E., Patel, A., Sheikh, A., Taylor, S.J., Standards for Reporting Implementation Studies (StaRI) Statement. BMJ, 2017. 356(i6795).

115. Sirriyeh R, Lawton R, Garner P, Armitage G. Reviewing studies with diverse designs: the development and evaluation of a new tool. J Eval Clin Pract. 2012;18:746-52.

116. Damschroder LJ, Aron DC, Keith RE, Kirsh SR, Alexander JA, Lowery JC. Fostering implementation of health services research findings into practice: a consolidated framework for advancing implementation science. Implement Sci. 2009;4(50):1-15.

117. Thomas J, Harden A. Methods for the thematic synthesis of qualitative research in systematic reviews. BMC Med Res Methodol. 2008;8(45):1-10,

118. Zasa S, Wright-De Aguero L, Briss PA, Truman BI, Hopkins DP, Hennessy MH, Sosin DM, Anderson L, Carande-Kulis V, Teutsch SM, Pappaioanou M. Data collection instrument and procedure for systematic reviews in the guide to community preventive services. Am J Prev Med. 2000;18(1S):44-74.

119. Segal N, Greenberg D, Dagan R, Ben-Shimol S. Disparities in PCV impact between different ethnic populations cohabiting the same region: a systematic review of the literature. Vaccine. 2016;34(37):4371-7.

120. Nicholls TR, Leach AJ, Morris PS. The short-term impact of each primary dose of pneumococcal conjugate vaccine on nasopharyngeal carriage: systematic review and meta-analyses of randimized controlled trials. Vaccine. 2016;34(6):703-213.

121. Graham S, Guy RJ, Cowie B, Wand HC, Donovan B, Akre SP, Ward JS, Chronic h B. Prevalence among aboriginal and Torres Strait islander Australians since universal vaccination: a systematic review and metaanalysis. BMC Infect Dis. 2013;13:403.

122. Grimshaw J, Thomas R, MacLennan G, Fraser C, Ramsay C, Vale L, Whitty P, Eccles M, Matowe L, Shirran L, Wensing M. Effectiveness and efficiency of guideline dissemination and implementation strategies. J Health Technol Assessment. 2004;8(6):1-94.

123. Baker, R., Camosso-Stefinovic, J., Gillies, C., Shaw, E.J., Cheater, F, Flottorp, S., Robertson, N., Tailored interventions to overcome identified barriers to change: effects on professional practice and health care outcomes. Cochrane Libr, 2010. Mar 2010.

124. Baker, R., Camosso-Stefinovic, J., Gillies, C., Shaw, E.J., Cheater, F, Flottorp, S., Robertson, N., Wensing, M., Fiander, M., Eccles, M., Godycki-Cwirko, M., Tailored interventions to address determinants of practice. Cochrane Libr, 2015. January 2015

125. Squires J, Sullivan K, Eccles M, Worswick J, Grimshaw J. Are multifaceted interventions more effective than single-compontent interventions in changing health-care professionals' behaviours? An overview of systematic reviews. Implement Sci. 2014;9(152):1-22.

126. Mehta P, Sharma M, Lee RC. Designing and evaluating a health belief model-based intervention to increase intent of HPV vaccination among college males. Int Q Community Health Educ. 2013-2014;34(1):101-17.

127. Paiva AL, Lipschitz JM, Fernandez AC, Redding CA, Prochaska JO. Evaluation of the acceptability and feasibility of a computer-tailored intevention to increase human papillomavirus vaccination among young adult women. J Am Coll Health. 2014:62(1):32-8.

128. Reiter PL, Stubbs B, Panozzo CA, Whitesell D, Brewer NT. HPV and HPV vaccine education intervention: effects on parents, healthcare staff, and school staff. Cancer Epidemiol Biomarkers Prev. 2011;2(11):2354-61. 
129. Kester LM, Shedd-Steel RB, Dotson-Roberts CA, Smith J, Zimet GD. The effects of a brief eduactional intervention on human papillomavirus knowledge and intention to initiate HPV vaccination in 18-26 year old young adults. Gynecol Oncol. 2014;132(supplement 1):S9-S12.

130. Perkins RB, Clark JA. What affects human papillomavirus vaccination rates? A qualitative analysis of providers' perceptions. Womens Health Issues. 2012;22(4):e379-86.

131. Rosenthal SL, Weiss TW, Zimet GD, Ma L, Good MB, Vichnin MD. Predictors of HPV vaccine uptake among women aged 19-26: importance of a physician's recommendation. Vaccine. 2011;29(5):890-5.

132. Sussman AL, Helitzer D, Sanders M, Urquieta B, Salvador M, Ndiaye K. HPV and cervical cancer prevention counselling with younger adolescents: implications for primary care. Ann Fam Med. 2007;5(4):298-304.

133. Thompson VLS, Arnold LD, Notaro SR. African American parents' attiudes toward HPV vaccination. Ethn Dis. 2011:21(3):335-41.

134. Gotvall M, Tyden T, Hoglund AT, Larsson M. Knowledge of human papillomavirus among high school students can be increased by an educational intervention. Int J STD AIDS. 2010;21(8):558-62.

135. Graham ID, Tetroe JM, McLean RK. In: Graham JMTID, Pearson A, editors. Chapter 1: some basics of integrated knowledge translation research in turning knowledge into action: practical guidance on how to do integrated knowledge translation research. Philadelphia, PA: Lippincott Williams \& Wilkins; 2014. p. 196

136. Canadian Institutes of Health Research (CIHR), Guide to Knowledge Translation Planning at CIHR: Integrated and End-of-Grant Approaches, K.T.a.P. Outreach, Editor. 2012, Her Majesty the Queen In Right of Canada: Ottawa, Canada.

137. Graham I, Logan J, Harrison MB, Straus SE, Tetroe J, Caswell W, Robinson N. Lost in knowledge translation: time for a map? J Contin Educ Heal Prof. 2006;26:13-24

138. Graham, I.D., \& Tetroe, J., and the KT Theories Research Group, Some theoretical underpinnings of knowledge translation. Acad Emerg Med, 2007. 14(936-941).

139. Moore J, Khan S, Straus S. Practising knowledge translation course - evidencebased, theory driven programs. In: Knowledge translation program (St. Michael's hospital). Toronto, ON, Canada: Toronto, Canada: Li Ka Shing Knowledge Institute of St. Michael's Hospital; 2015.

140. Michie S, van Stralen M, West R. The behaviour change wheel: a new method of characterising and designing behaviour change interventions. Implement Sci. 2011:6(42):1-12.

141. Michie S, Atkins L, West R, editors. The behaviour change wheel: a guide to designing interventions. First ed. Great Britain: Silverback Publishing; 2014. p. 329.

142. The Guide to Community Preventive Services (The Community Guide). The guide to community preventive services. 2015 [cited 2017 February 10, 2017]; Available from: https://www.thecommunityguide.org/about/ourmethodology.

\section{Submit your next manuscript to BioMed Central and we will help you at every step:}

- We accept pre-submission inquiries

- Our selector tool helps you to find the most relevant journal

- We provide round the clock customer support

- Convenient online submission

- Thorough peer review

- Inclusion in PubMed and all major indexing services

- Maximum visibility for your research

Submit your manuscript at www.biomedcentral.com/submit

CBiomed Central 